\title{
Outlier Detection in Adaptive Functional-Coefficient Autoregressive Models Based on Extreme Value Theory
}

\author{
Ping Chen, ${ }^{1}$ Ling Dong, ${ }^{2}$ Wanyi Chen, ${ }^{3}$ and Jin-Guan Lin ${ }^{1}$ \\ ${ }^{1}$ Department of Mathematics, Southeast University, Nanjing, Jiangsu 210096, China \\ ${ }^{2}$ School of Finance and Statistics, East China Normal University, Shanghai 200241, China \\ ${ }^{3}$ Department of Industrial Engineering and Operations Research, Columbia University, New York, NY 10027, USA
}

Correspondence should be addressed to Jin-Guan Lin; jglin@seu.edu.cn

Received 26 January 2013; Accepted 12 March 2013

Academic Editor: Ming Li

Copyright (C) 2013 Ping Chen et al. This is an open access article distributed under the Creative Commons Attribution License, which permits unrestricted use, distribution, and reproduction in any medium, provided the original work is properly cited.

\begin{abstract}
This paper proposes several test statistics to detect additive or innovative outliers in adaptive functional-coefficient autoregressive (AFAR) models based on extreme value theory and likelihood ratio tests. All the test statistics follow a tractable asymptotic Gumbel distribution. Also, we propose an asymptotic critical value on a fixed significance level and obtain an asymptotic $p$-value for testing, which is used to detect outliers in time series. Simulation studies indicate that the extreme value method for detecting outliers in AFAR models is effective both for AO and IO, for a lone outlier and multiple outliers, and for separate outliers and outlier patches. Furthermore, it is shown that our procedure can reduce possible effects of masking and swamping.
\end{abstract}

\section{Introduction}

Outlier detection and analysis play important roles in practical applications. For instance, outlier detection can be applied to anomaly detection in computer networks, financial time series, and data series in geosciences, as can be seen from [1, Chap. 1] and [2]. Other examples include the study on loss of customers in the commercial field and the detection and tracking of financial crime as credit card fraud, all of which involve and exploit the useful information provided by the presence of outliers. On the other hand, outliers in dynamic systems or engineering time series [3] can have adverse effects on model identification and parameter estimation, where eliminating outliers is necessary in the statistical modeling of time series for the purpose of preprocessing data, see, for example, [4]. Some studies have even shown that the emerging of outliers generates certain nonlinear time series. Several procedures are available in the literature to deal with problems related to outliers. Chen [5] developed a method for detecting additive outliers in bilinear time series, which belong to the family of fractal time series models [6]. Cai et al. [7] studied the functional-coefficient regression models of nonlinear time series. Battaglia [8] discovered a way to identify and estimate outliers in functional autoregressive time series. Battaglia and Orfei [9] addressed the issue of outlier detection and estimation in nonlinear time series. Chen et al. [10] and Chen et al. [11] discussed the detection of outlier patches, change point, and outliers in bilinear models.

Extreme value theory and likelihood ratio tests have been used in the detection of outliers and time series analysis. For instance, Martin [12] conducted extreme value analysis on the optimal level cross-prediction of linear Gaussian processes. Zhu and Ling [13] performed likelihood ratio tests on the structural change from an $A R(p)$ model to a threshold $\mathrm{AR}(\mathrm{p})$ model. Furthermore, based on extreme value theory Chareka et al. [14] proposed an alternative test method for the detection of additive outliers in Gaussian time series. On the other hand, some scholars such as Fung et al. [15] and Río [16], focused their studies on special cases of outliers detection. It is commonly agreed that the key of outlier detection lies in determining whether the test statistic exceeds a critical value, that is, the threshold under a given significance level. However, explanations for the selection of threshold in many literatures are ambiguous, and the threshold itself can hardly be controlled under a certain level of significance. In this paper, we propose an asymptotic 
critical value on a fixed significance level, which is used to detect additive and innovative outliers in adaptive functionalcoefficient autoregressive (AFAR) models (see, e.g., Fan and Yao [17]).

This paper is structured as follows. In Section 2, we consider AFAR models with additive or innovative outliers and their estimation. In Section 3, several procedures are proposed to detect outliers in the AFAR models based on extreme value theory and likelihood ratio tests. In Section 4, we present some simulation studies and demonstrate the effectiveness of the proposed method through an empirical way. Concluding remarks are summarized in Section 5.

\section{Outliers Models and Test Statistics}

We now consider the AFAR model:

$$
\begin{aligned}
x_{t}= & \frac{\cos \left(a_{1} x_{t-2}\right)}{a_{2}+a_{3} x_{t-2}^{2}} x_{t-1} \\
& +\left(a_{4}+a_{5} \exp \left(a_{6} x_{t-2}^{2}\right)\right) x_{t-2}+\varepsilon_{t},
\end{aligned}
$$

which can be written as

$$
x_{t}=\sum_{j=1}^{2} f_{j}\left(x_{t-2}\right) x_{t-j}+\varepsilon_{t},
$$

where $\varepsilon_{t}$ is a Gaussian white noise with mean zero and variance $\sigma^{2}$ and $f_{1}\left(x_{t-2}\right)=\cos \left(a_{1} x_{t-2}\right) /\left(a_{2}+a_{3} x_{t-2}^{2}\right), f_{2}\left(x_{t-2}\right)=$ $a_{4}+a_{5} \exp \left(a_{6} x_{t-2}^{2}\right)$.

Suppose that $\left\{x_{t}\right\}_{t=1}^{n}$ is a zero-mean stationary process following model (2) description, and there is an outlier at time $t=q$, whose influence magnitude is $\omega_{q}$, then the observed series $\left\{y_{t}\right\}$ may be presented as follows.

(1) Innovative outlier (IO) model:

$$
\begin{aligned}
& y_{t}=x_{t}, \quad 1 \leq t<q \\
& y_{t}=\sum_{j=1}^{2} f_{j}\left(y_{t-2}\right) y_{t-j}+\eta_{t}, \quad q \leq t \leq n, \\
& \eta_{t}=\varepsilon_{t}+\omega_{q}^{\mathrm{IO}} \cdot \delta(t-q) .
\end{aligned}
$$

(2) Additive outlier ( $\mathrm{AO})$ model:

$$
y_{t}=x_{t}+\omega_{q}^{\mathrm{AO}} \cdot \delta(t-q), \quad t=1, \ldots, n,
$$

where $\delta(t)$ is the Kronecker symbol: If $t=0$, then $\delta(t)=1$, else $\delta(t)=0$.

It is obvious that the detection for IO or AO may be transformed to test whether the null hypothesis $H_{0}: \omega_{q}^{\mathrm{IO}}=0$ or $H_{0}^{\prime}: \omega_{q}^{\mathrm{AO}}=0$ is true under a certain level of significance. This can be solved by knowing the distributions of $\widehat{\omega}_{q}^{\mathrm{IO}}$ and $\widehat{\omega}_{q}^{\mathrm{AO}}$ under the null hypothesis. We estimate $\omega_{q}$ by using the maximum likelihood method. The maximum likelihood function of observations is the positive ratio of the maximum likelihood function of residuals. For a given $q$, assume that the $f_{j}$ and corresponding parameters are known, then we can obtain the estimation of $\omega_{q}$ by maximizing the likelihood function. For convenience, suppose that there is no outlier in the two former observations. Denote the residual of observations by $\eta_{t}$, for any $k \leq 0$, and let $\varepsilon_{k}=0, x_{k}=$ 0 , and $f_{k}(\cdot)=0 . \varepsilon_{1}, \varepsilon_{2}$ be obtained by letting $\varepsilon_{t}=x_{t}-$ $\sum_{j=1}^{2} f_{j}\left(x_{t-2}\right) x_{t-j}$. Under initial conditions mentioned above, the conditional likelihood function of $\omega_{q}$ is given by

$$
\begin{aligned}
l(y \mid \omega)=l(x)=l(\varepsilon) & =\prod_{t=3}^{n}\left(2 \pi \sigma^{2}\right)^{-1 / 2} \exp \left(-\frac{\varepsilon_{t}^{2}}{2 \sigma^{2}}\right) \\
& =\left(2 \pi \sigma^{2}\right)^{-(n-2) / 2} \exp \left(-\frac{\sum_{t=3}^{n} \varepsilon_{t}^{2}}{2 \sigma^{2}}\right) .
\end{aligned}
$$

We can obtain the maximum likelihood estimation of $\omega_{q}$ at the minimum of $\sum_{t=3}^{n} \varepsilon_{t}^{2}$. It is an accurate estimation for the linear model. However, it is just an approximation for the nonlinear model. For $t>2$, the residual of observations is given by $\eta_{t}=y_{t}-\sum_{j=1}^{2} f_{j}\left(y_{t-2}\right) y_{t-j}$. We then discuss the estimations of $\omega_{q}^{\mathrm{IO}}$ and $\omega_{q}^{\mathrm{AO}}$ for model (2).

(1) Assume that there is an IO at $t=q$ and its influence magnitude is $\omega_{q}^{\mathrm{IO}}$. From (3), it follows that $\eta_{q}=\varepsilon_{q}+\omega_{q}^{\mathrm{IO}}$ and $\eta_{t}=\varepsilon_{t}(t \neq q)$, where $\sum_{t=3}^{n} \varepsilon_{t}^{2}=\sum_{t \neq q} \eta_{t}^{2}+\left(\eta_{q}-\omega_{q}^{\mathrm{IO}}\right)^{2}$. So, the maximum likelihood estimation of $\omega_{q}^{\text {IO }}$ is given by

$$
\widehat{\omega}_{q}^{\mathrm{IO}}=\eta_{q}=y_{q}-\sum_{j=1}^{2} f_{j}\left(y_{q-2}\right) y_{q-j} .
$$

For known $f_{j}$ and $\sigma^{2}$, we have $\widehat{\omega}_{q}^{\mathrm{IO}} \sim N\left(\omega_{q}^{\mathrm{IO}}, \sigma^{2}\right)$, which is similar to Battaglia [8]. Under the null hypothesis $H_{0}: \omega_{q}^{\mathrm{IO}}=$ $0, \widehat{\omega}_{q}^{\mathrm{IO}} \sim N\left(0, \sigma^{2}\right)$, we obtain likelihood radio test statistic $\hat{\lambda}_{q}^{\mathrm{IO}}=\left(\widehat{\omega}_{q}^{\mathrm{IO}} / \sigma\right) \sim N(0,1)$ by standardizing $\widehat{\omega}_{q}^{\mathrm{IO}}$. When $f_{j}$ and $\sigma^{2}$ are unknown, they can be replaced by their consistent estimations $\widehat{\sigma}_{\mathrm{IO}}^{2}=(n-2)^{-1} \sum_{t=3, t \neq q}^{n} \widehat{\eta}_{t}^{2}$ and $\hat{\eta}_{t}=y_{t}-$ $\sum_{j=1}^{2} \hat{f}_{j}\left(y_{t-2}\right) y_{t-j}$. Similarly, we also have that

$$
\widehat{\lambda}_{q}^{\mathrm{IO}}=\frac{\widehat{\omega}_{q}^{\mathrm{IO}}}{\widehat{\sigma}_{\mathrm{IO}}} \sim N(0,1) .
$$

(2) Assume that there is an $\mathrm{AO}$ at $t=q$ and its influence magnitude is $\omega_{q}^{\mathrm{AO}}$. From (4), it follows that

$$
\begin{aligned}
\eta_{q} & =y_{q}-\sum_{j=1}^{2} f_{j}\left(y_{q-2}\right) y_{q-j} \\
& =x_{q}+\omega_{q}^{\mathrm{AO}}-\sum_{j=1}^{2} f_{j}\left(x_{q-2}\right) x_{q-j} \\
& =\varepsilon_{q}+\omega_{q}^{\mathrm{AO}} .
\end{aligned}
$$


When $t<q, \eta_{t}=y_{t}-\sum_{j=1}^{2} f_{j}\left(y_{t-2}\right) y_{t-j}=x_{t}-$ $\sum_{j=1}^{2} f_{j}\left(x_{t-2}\right) x_{t-j}=\varepsilon_{t}$, if $t>q$, that is, $k>0$, then $\eta_{q+k}=$ $y_{q+k}-\sum_{j=1}^{2} f_{j}\left(y_{q+k-2}\right) y_{q+k-j}$, if $k=1$, we have that

$$
\begin{aligned}
\eta_{q+1} & =y_{q+1}-\sum_{j=1}^{2} f_{j}\left(y_{q-1}\right) y_{q+1-j} \\
& =x_{q+1}-\sum_{j=1}^{2} f_{j}\left(x_{q-1}\right)\left(x_{q+1-j}+\omega_{q}^{\mathrm{AO}} \delta(1-j)\right) \\
& =\varepsilon_{q+1}-\sum_{j=1}^{2} f_{j}\left(x_{q-1}\right) \omega_{q}^{\mathrm{AO}} \delta(1-j) \\
& =\varepsilon_{q+1}-f_{1}\left(x_{q-1}\right) \omega_{q}^{\mathrm{AO}}=\varepsilon_{q+1}-f_{1}\left(y_{q-1}\right) \omega_{q}^{\mathrm{AO}},
\end{aligned}
$$

and if $k=2$, we have that

$$
\begin{aligned}
\eta_{q+2}= & y_{q+2}-\sum_{j=1}^{2} f_{j}\left(y_{q}\right) y_{q+2-j} \\
= & x_{q+2}-\sum_{j=1}^{2} f_{j}\left(x_{q}\right) x_{q+2-j}+\sum_{j=1}^{2} f_{j}\left(x_{q}\right) x_{q+2-j} \\
& -\sum_{j=1}^{2} f_{j}\left(y_{q}\right) y_{q+2-j} \\
= & \varepsilon_{q+2}-\left(f_{1}\left(y_{q}\right)-f_{1}\left(x_{q}\right)\right) y_{q+1} \\
& +f_{2}\left(x_{q}\right)\left(y_{q}-\omega_{q}^{\mathrm{AO}}\right)-f_{2}\left(y_{q}\right) y_{q} \\
= & \varepsilon_{q+2}-f_{1}^{\prime}\left(x_{q}+\theta_{1} \omega_{q}^{\mathrm{AO}}\right) \omega_{q}^{\mathrm{AO}} y_{q+1} \\
& -\left(f_{2}\left(y_{q}\right)-f_{2}\left(x_{q}\right)\right) y_{q}-f_{2}\left(x_{q}\right) \omega_{q}^{\mathrm{AO}} \\
= & \varepsilon_{q+2}-f_{1}^{\prime}\left(x_{q}+\theta_{1} \omega_{q}^{\mathrm{AO}}\right) \omega_{q}^{\mathrm{AO}} y_{q+1} \\
& -f_{2}^{\prime}\left(x_{q}+\theta_{2} \omega_{q}^{\mathrm{AO}}\right) \omega_{q}^{\mathrm{AO}} y_{q}-f_{2}\left(x_{q}\right) \omega_{q}^{\mathrm{AO}} \\
= & \varepsilon_{q+2}-\omega_{q}^{\mathrm{AO}} \cdot\left[f_{2}\left(x_{q}\right)+\sum_{j=1}^{2} f_{j}^{\prime}\left(x_{q, j}^{*}\right) y_{q+2-j}\right],
\end{aligned}
$$

where $x_{q, j}^{*}=x_{q}+\theta_{j} \omega_{q}^{\mathrm{AO}}, 0<\theta_{j}<1$, and $j=1$, 2. If $k>2$, then $\eta_{q+k}=\varepsilon_{q+k}$, from which it follows that

$$
\eta_{q+k}=c_{k} \omega_{q}^{\mathrm{AO}}+\varepsilon_{q+k}, \quad k=1,2,
$$

where $c_{1}=-f_{1}\left(y_{q-1}\right)$ and $c_{2}=-\left[f_{2}\left(x_{q}\right)+\sum_{j=1}^{2} f_{j}^{\prime}\left(x_{q, j}^{*}\right) y_{q+2-j}\right]$, so we have that

$$
\begin{aligned}
\sum_{t=3}^{n} \varepsilon_{t}^{2} & =\sum_{t=3}^{q-1} \varepsilon_{t}^{2}+\varepsilon_{q}^{2}+\sum_{t=q+1}^{q+2} \varepsilon_{t}^{2}+\sum_{t=q+3}^{n} \varepsilon_{t}^{2} \\
& =\sum_{t=3}^{q-1} \eta_{t}^{2}+\left(\eta_{q}-\omega_{q}^{\mathrm{AO}}\right)^{2}+\sum_{k=1}^{2}\left(\eta_{q+k}-c_{k} \omega_{q}^{\mathrm{AO}}\right)^{2}+\sum_{t=q+3}^{n} \eta_{t}^{2}
\end{aligned}
$$

$$
\begin{aligned}
= & \sum_{t=3}^{q-1} \eta_{t}^{2}+\sum_{t=q+3}^{n} \eta_{t}^{2}+\left(1+c_{1}^{2}+c_{2}^{2}\right) \omega_{q}^{\mathrm{AO}^{2}} \\
& -2\left(\eta_{q}+c_{1} \eta_{q+1}+c_{2} \eta_{q+2}\right) \omega_{q}^{\mathrm{AO}}+\left(\eta_{q}^{2}+\eta_{q+1}^{2}+\eta_{q+2}^{2}\right) .
\end{aligned}
$$

By minimizing the above expression, we obtain

$$
\omega_{q}^{\mathrm{AO}}=\frac{\eta_{q}+c_{1} \eta_{q+1}+c_{2} \eta_{q+2}}{1+c_{1}^{2}+c_{2}^{2}},
$$

where $c_{1}$ may be obtained by estimation of $-f_{1}\left(y_{q-1}\right)$, while $c_{2}$ is complicated and difficult to be confirmed. Nevertheless, Battaglia [8] indicated that we could estimate $c_{2}$ by $-f_{2}\left(y_{q}\right)$, which is convenient and effective. If all $f_{j}(\cdot)$ and $\sigma^{2}$ are known, then we have

$$
\begin{aligned}
& \widehat{\omega}_{q}^{\mathrm{AO}}=\frac{\eta_{q}-f_{1}\left(y_{q-1}\right) \eta_{q+1}-f_{2}\left(y_{q}\right) \eta_{q+2}}{\Delta_{q}}, \\
& \Delta_{q}=1+f_{1}^{2}\left(y_{q-1}\right)+f_{2}^{2}\left(y_{q}\right) .
\end{aligned}
$$

Similar to Battaglia [8], we obtain

$$
\widehat{\omega}_{q}^{\mathrm{AO}} \sim N\left(\omega_{q}^{\mathrm{AO}}, \frac{\sigma^{2}}{\Delta_{q}}\right) .
$$

Under the null hypothesis $H_{0}^{\prime}: \omega_{q}^{\mathrm{AO}}=0$, we obtain likelihood radio test statistics $\hat{\lambda}_{q}^{\mathrm{AO}}=\left(\widehat{\omega}_{q}^{\mathrm{AO}} /\left(\sigma / \sqrt{\Delta_{q}}\right)\right) \sim N(0,1)$ by standardizing $\widehat{\omega}_{q}^{\mathrm{AO}}$. In practice, if $f_{j}(\cdot)$ and $\sigma^{2}$ are unknown, which can be replaced by their consistent estimations $\widehat{\sigma}_{\mathrm{AO}}^{2}=(n-$ $2)^{-1}\left[\sum_{t=3}^{n} \widehat{\eta}_{t}^{2}-\left(\widehat{\omega}_{q}^{\mathrm{AO}}\right)^{2} \cdot \sum_{j=1}^{2} \widehat{f}_{j}^{2}\left(y_{q+j-2}\right)\right]$, we likewise obtain

$$
\hat{\lambda}_{q}^{\mathrm{AO}}=\frac{\widehat{\omega}_{q}^{\mathrm{AO}}}{\widehat{\sigma}_{\mathrm{AO}} / \sqrt{\widehat{\widehat{\Delta}}_{q}}} \sim N(0,1) .
$$

It is indicated by (6) and (13) that the best estimate of $\omega$ is $\eta_{q}$ at time $q$ for the case of IO. the best estimate of $\omega$ is the linear combination of errors $\eta_{q}, \eta_{q+1}$, and $\eta_{q+2}$ at time $q$ in the case of AO. If the location $q$ of outlier is known, the distributions of likelihood ratio test statistics $\hat{\lambda}_{q}^{\mathrm{IO}}$ and $\hat{\lambda}_{q}^{\mathrm{AO}}$ are given by (7) and (16). However, in practice the location of outlier is unknown, thus we should inspect the magnitude of test statistic at every time point. By replacing $\hat{\lambda}_{q}^{\mathrm{IO}}$ and $\hat{\lambda}_{q}^{\mathrm{AO}}$ with $\hat{\lambda}_{t}^{\mathrm{IO}}$ and $\hat{\lambda}_{t}^{\mathrm{AO}}$, the following theorem indicates that the processes of $\left\{\hat{\lambda}_{t}^{\mathrm{IO}}\right\}$ and $\left\{\hat{\lambda}_{t}^{\mathrm{AO}}\right\}$ are both Gaussian process, respectively.

Theorem 1. (1) Under the hypothesis $H_{0}: \omega_{t}^{\mathrm{IO}}=0$, that is, there is no IO at any time, $\left\{\widehat{\omega}_{t}^{\mathrm{IO}}\right\}$ is a stationary zero-mean Gaussian process with variance $\sigma^{2}$, and its autocovariance function is $r^{\mathrm{IO}}(h)=\sigma^{2} \cdot I(h=0)$, where $I_{A}(x)$ is the index function. If $x \in A$, then $I_{A}(x)=1$, and else $I_{A}(x)=0$. 
(2) Under the hypothesis $H_{0}^{\prime}: \omega_{t}^{\mathrm{AO}}=0$, that is, there is no $\mathrm{AO}$ at any time, $\left\{\widehat{\omega}_{t}^{\mathrm{AO}}\right\}$ is a stationary zero-mean Gaussian process with variance $\sigma^{2} / \Delta_{t}$, and its autocovariance function is

$$
\begin{aligned}
r^{\mathrm{AO}}(t, h)=\sigma^{2} \Delta_{t}^{-1} \Delta_{t+h}^{-1} & {\left[\sum_{j=h+1}^{2} f_{j}\left(y_{t+j-2}\right) f_{j-h}\left(y_{t+j-2}\right)\right.} \\
& \left.-f_{h}\left(y_{t+h-2}\right)\right] I \quad(1 \leq h \leq 2) .
\end{aligned}
$$

Proof. The Gaussian character is shown by (7) and (16). We derive their autocovariance function as follows.

(1) Under the null hypothesis $H_{0}: \omega_{t}^{\mathrm{IO}}=0$, we have $\eta_{t}=$ $\varepsilon_{t}$ and

$$
\begin{aligned}
r^{\mathrm{IO}}(h) & =\operatorname{cov}\left(\widehat{\omega}_{t}^{\mathrm{IO}}, \widehat{\omega}_{t+h}^{\mathrm{IO}}\right)=\operatorname{cov}\left(\eta_{t}, \eta_{t+h}\right) \\
& =\operatorname{cov}\left(\varepsilon_{t}, \varepsilon_{t+h}\right)=\sigma^{2} \cdot I \quad(h=0) .
\end{aligned}
$$

(2) Under the null hypothesis $H_{0}^{\prime}: \omega_{t}^{\mathrm{AO}}=0$, we have $\eta_{t}=\varepsilon_{t}$ and

$$
\begin{aligned}
& r^{\mathrm{AO}}(t, h)=\operatorname{cov}\left(\widehat{\omega}_{t}^{\mathrm{AO}}, \widehat{\omega}_{t+h}^{\mathrm{AO}}\right) \\
& =\operatorname{cov}\left(\Delta_{t}^{-1}\left(\eta_{t}-\sum_{j=1}^{2} f_{j}\left(y_{t+j-2}\right) \eta_{t+j}\right),\right. \\
& \left.\Delta_{t+h}^{-1}\left(\eta_{t+h}-\sum_{j=1}^{2} f_{j}\left(y_{t+h+j-2}\right) \eta_{t+h+j}\right)\right) \\
& =\Delta_{t}^{-1} \Delta_{t+h}^{-1}\left[\operatorname{cov}\left(\eta_{t}, \eta_{t+h}\right)\right. \\
& -\sum_{j=1}^{2} f_{j}\left(y_{t+h+j-2}\right) \operatorname{cov}\left(\eta_{t}, \eta_{t+h+j}\right) \\
& -\sum_{j=1}^{2} f_{j}\left(y_{t+j-2}\right) \operatorname{cov}\left(\eta_{t+j}, \eta_{t+h}\right) \\
& +\operatorname{cov}\left(\sum_{j=1}^{2} f_{j}\left(y_{t+j-2}\right) \eta_{t+j}\right. \\
& \left.\left.\sum_{j=1}^{2} f_{j}\left(y_{t+h+j-2}\right) \eta_{t+h+j}\right)\right] \\
& =\sigma^{2} \Delta_{t}^{-1} \Delta_{t+h}^{-1}\left[\sum_{j=h+1}^{2} f_{j}\left(y_{t+j-2}\right) f_{j-h}\left(y_{t+j-2}\right)\right. \\
& \left.-f_{h}\left(y_{t+h-2}\right)\right] I \quad(1 \leq h \leq 2) \text {. }
\end{aligned}
$$

We know that $\left\{\widehat{\omega}_{t}^{\mathrm{IO}}\right\}$ is not only Gaussian, but also a stationary process by Theorem 1 . Also, it is serial independent under the null hypothesis $H_{0}: \omega_{t}^{\mathrm{IO}}=0$. However, despite that $\left\{\widehat{\omega}_{t}^{\mathrm{AO}}\right\}$ is Gaussian, it is not stationary due to the fact that its autocovariance function $r^{\mathrm{AO}}(t, h)$ is dependent on $t$. Besides, $\left\{\widehat{\omega}_{t}^{\mathrm{AO}}\right\}$ is truncated in finite steps, which means that its maximum correlation is within two steps; that is, $\widehat{\omega}_{t}^{\mathrm{AO}}$ is independent with $\widehat{\omega}_{t+h}^{\mathrm{AO}}$ when $h>2$. By standardizing $\left\{\widehat{\omega}_{t}^{\mathrm{IO}}\right\}$ and $\left\{\widehat{\omega}_{t}^{\mathrm{AO}}\right\}$, we can obtain $\left\{\widehat{\lambda}_{t}^{\mathrm{IO}}\right\}$ and $\left\{\widehat{\lambda}_{t}^{\mathrm{AO}}\right\}$. It is obvious that $\left\{\hat{\lambda}_{t}^{\mathrm{IO}}\right\}$ is a standardized stationary Gaussian process under the hypothesis $H_{0}: \omega_{t}^{\mathrm{IO}}=0$. Also, $\left\{\hat{\lambda}_{t}^{\mathrm{AO}}\right\}$ is a standardized Gaussian process under the hypothesis $H_{0}^{\prime}: \omega_{t}^{\mathrm{AO}}=0$, but it is not stationary, whereas its autocovariance function is truncated in finite steps.

\section{Detection of Outliers Based on Extreme Value Theory}

As similar to Chareka et al. [14] and Leadbetter and Rootzén [18], we obtain the following.

Lemma 2 (see [18]). Assume that $\left\{X_{n}\right\}$ is a stationary zeromean Gaussian time series and its autocorrelation function is $\rho(h)$. Let $Z_{n}=\max \left\{X_{1}, X_{2}, \ldots, X_{n}\right\}$, and if the Berman condition $\lim _{h \rightarrow \infty} \rho(h) \cdot \log h=0$ is satisfied, then one has that $P\left(\left(\left(Z_{n}-c_{n}\right) / d_{n}\right) \leq x\right) \rightarrow \Lambda(x)=\exp \left\{-e^{-x}\right\}, n \rightarrow \infty$, where $d_{n}=(2 \log n)^{-1 / 2}$, and $c_{n}=\left(1 / d_{n}\right)-\left(d_{n} / 2\right)(\log \log n+$ $\log (4 \pi))$.

Lemma 3 (see [18]). Assume that $\left\{X_{n}\right\}$ is a stationary zeromean Gaussian time series, and let $A_{n}=\max _{t=1}^{n}\left\{\left|X_{t}\right|\right\}$ and $Z_{2 n}=\max _{t=1}^{2 n}\left\{X_{t}\right\}$, and if $X_{n}$ satisfies the Berman condition, then $A_{n}$ and $Z_{2 n}$ have the same distribution.

Theorem 4. Let $\eta_{\mathrm{IO}}=\max _{t=1,2, \ldots, n}\left\{\left|\widehat{\lambda}_{t}^{\mathrm{IO}}\right|\right\}$ and $\eta_{\mathrm{AO}}=$ $\max _{t=1,2, \ldots, n}\left\{\left|\hat{\lambda}_{t}^{\mathrm{AO}}\right|\right\}$, and under the hypothesis $H_{0}: \omega_{t}^{\mathrm{IO}}=0$ and $H_{0}^{\prime}: \omega_{t}^{\mathrm{AO}}=0$, one has that

$$
\begin{gathered}
P\left(\frac{\eta_{\mathrm{IO}}-c_{2 n}}{d_{2 n}} \leq x\right) \rightarrow \Lambda(x), \\
P\left(\frac{\eta_{\mathrm{AO}}-c_{2 n}}{d_{2 n}} \leq x\right) \rightarrow \Lambda(x), \quad n \rightarrow \infty,
\end{gathered}
$$

where $d_{2 n}=[2 \log (2 n)]^{-1 / 2}$ and $c_{2 n}=\left(1 / d_{2 n}\right)-\left(d_{2 n} /\right.$ 2) $[\log \log (2 n)+\log (4 \pi)]$.

Proof. It follows from Theorem 1 that the autocorrelation function of $\left\{\hat{\lambda}_{t}^{\mathrm{IO}}\right\}$ is $\rho^{\mathrm{IO}}(h)=I(h=0)$ under the hypothesis $H_{0}: \omega_{t}^{\mathrm{IO}}=0$, so $\lim _{h \rightarrow \infty} \rho^{\mathrm{IO}}(h) \cdot \log h=0$; that is, the Berman condition is satisfied. Again, $\left\{\hat{\lambda}_{t}^{\mathrm{IO}}\right\}$ is a stationary zeromean Gaussian time series. Hence, from Lemma 2, we have that $P\left(\left(\left(\max _{t=1}^{n}\left\{\hat{\lambda}_{t}^{\mathrm{IO}}\right\}-c_{n}\right) / d_{n}\right) \leq x\right) \rightarrow \Lambda(x)$. Again from Lemma 3, we know that the distribution of $\eta_{\mathrm{IO}}=$ $\max _{t=1}^{n}\left\{\left|\hat{\lambda}_{t}^{\mathrm{IO}}\right|\right\}$ is the same as that of $\max _{t=1}^{2 n}\left\{\hat{\lambda}_{t}^{\mathrm{IO}}\right\}$, so we 
have that $P\left(\left(\left(\eta_{\mathrm{IO}}-c_{2 n}\right) / d_{2 n}\right) \leq x\right) \rightarrow \Lambda(x)$. Similarly, under the hypothesis $H_{0}^{\prime}: \omega_{t}^{\mathrm{AO}}=0$, from Theorem 1 we know that the autocorrelation function of $\hat{\lambda}_{t}^{\mathrm{AO}}$ is

$$
\begin{aligned}
\rho^{\mathrm{AO}}(t, h)=\Delta_{t}^{-1 / 2} \cdot \Delta_{t+h}^{-1 / 2} \cdot & {\left[\sum_{j=h+1}^{2} f_{j}\left(y_{t+j-2}\right) f_{j-h}\left(y_{t+j-2}\right)\right.} \\
& \left.-f_{h}\left(y_{t+h-2}\right)\right] I \quad(1 \leq h \leq 2),
\end{aligned}
$$

where $\lim _{h \rightarrow \infty} \rho^{\mathrm{AO}}(h) \cdot \log h=0$; that is, the Berman condition is satisfied. Therefore, similar to the statements above, it must hold that $P\left(\left(\left(\eta_{\mathrm{AO}}-c_{2 n}\right) / d_{2 n}\right) \leq x\right) \rightarrow$ $\Lambda(x)$.

Lemma 5 (see [14]). Assume that $\left\{X_{n}\right\}$ is a stationary zeromean Gaussian time series with variance 1 and autocorrelation function $\rho(h)$, and let $M_{n}=\max \left\{X_{1}^{2}, \ldots, X_{n}^{2}\right\}$, and if $\rho(h)$ satisfies the Berman condition, then one has $P\left(\left(\left(M_{n}-e_{n}\right) / 2\right) \leq\right.$ $x) \rightarrow \Lambda(x), n \rightarrow \infty$, where $e_{n}=2 \log n-\log (\log n)-\log \pi$.

Theorem 6. Let $\vartheta_{\mathrm{IO}}=\max _{t=1}^{n}\left\{\left(\hat{\lambda}_{t}^{\mathrm{IO}}\right)^{2}\right\}$ and $\vartheta_{\mathrm{AO}}=$ $\max _{t=1}^{n}\left\{\left(\hat{\lambda}_{t}^{\mathrm{AO}}\right)^{2}\right\}$, under the hypothesis $H_{0}: \omega_{t}^{\mathrm{IO}}=0$ and $H_{0}^{\prime}: \omega_{t}^{\mathrm{AO}}=0$, and if the corresponding Berman conditions for $\left\{\hat{\lambda}_{t}^{\mathrm{IO}}\right\}$ and $\left\{\hat{\lambda}_{t}^{\mathrm{AO}}\right\}$ are satisfied, then one has

$$
\begin{gathered}
P\left(\frac{\vartheta_{\mathrm{IO}}-e_{n}}{2} \leq x\right) \rightarrow \Lambda(x), \\
P\left(\frac{\vartheta_{\mathrm{AO}}-e_{n}}{2} \leq x\right) \rightarrow \Lambda(x), \quad n \rightarrow \infty,
\end{gathered}
$$

where $e_{n}=2 \log n-\log (\log n)-\log \pi$.

Proof. We know that the Berman conditions for $\left\{\lambda_{t}^{\mathrm{IO}}\right\}$ and $\left\{\lambda_{t}^{\mathrm{AO}}\right\}$ are satisfied under the hypothesis $H_{0}: \omega_{t}^{\mathrm{IO}}=0$ and $H_{0}^{\prime}: \omega_{t}^{\mathrm{AO}}=0$ by the inferential process of Theorem 4 . Thus, the conclusion is also obtained by Lemma 5 .

Let $\alpha$ be the test significance level and $y_{n}=(1+(1-$ $\left.\alpha)^{1 / n}\right) / 2$. We denote the $t$-distribution function with $n$ degrees of freedom by $H_{n}(t)$. Let $x_{n}=H_{n-2}^{-1}\left(y_{n}\right)$ and $\bar{e}_{n}=(n /(n-$ 1)) $x_{n}^{2}-2 \Lambda^{-1}(1-\alpha)$, where $\Lambda^{-1}(1-\alpha)$ is the $1-\alpha$ quantile of the Gumbel distribution. Similarly, we have the following.

Theorem 7. Under the conditions above, one has that

$$
\begin{gathered}
P\left(\frac{M_{n}-\bar{e}_{n}}{2} \leq x\right) \rightarrow \Lambda(x), \\
P\left(\frac{\vartheta_{\mathrm{IO}}-\bar{e}_{n}}{2} \leq x\right) \rightarrow \Lambda(x), \\
P\left(\frac{\vartheta_{\mathrm{AO}}-\bar{e}_{n}}{2} \leq x\right) \rightarrow \Lambda(x), \quad n \rightarrow \infty .
\end{gathered}
$$

At this point, it is convenient to introduce two more pieces of notation: $A_{t}^{\mathrm{IO}}=\left(\left(\left|\hat{\lambda}_{t}^{\mathrm{IO}}\right|-c_{2 n}\right) / d_{2 n}\right)$ and $A_{t}^{\mathrm{AO}}=$ $\left(\left(\left|\hat{\lambda}_{t}^{\mathrm{AO}}\right|-c_{2 n}\right) / d_{2 n}\right)$, which we call absolute value test statistics; $S_{t}^{\mathrm{IO}}=\left(\left(\left(\hat{\lambda}_{t}^{\mathrm{IO}}\right)^{2}-e_{n}\right) / 2\right)$ and $S_{t}^{\mathrm{AO}}=\left(\left(\left(\hat{\lambda}_{t}^{\mathrm{AO}}\right)^{2}-e_{n}\right) / 2\right)$, which we call square test statistics; $C_{t}^{\mathrm{IO}}=\left(\left(\left(\hat{\lambda}_{t}^{\mathrm{IO}}\right)^{2}-\bar{e}_{n}\right) / 2\right)$ and $C_{t}^{\mathrm{AO}}=\left(\left(\left(\hat{\lambda}_{t}^{\mathrm{AO}}\right)^{2}-\bar{e}_{n}\right) / 2\right)$, which we call adjusted square test statistic. We likewise obtain

$$
\begin{gathered}
P\left(\max _{t=1}^{n}\left\{A_{t}^{\mathrm{IO}}\right\} \leq x\right) \rightarrow \Lambda(x), \\
P\left(\max _{t=1}^{n}\left\{A_{t}^{\mathrm{AO}}\right\} \leq x\right) \rightarrow \Lambda(x), \\
P\left(\max _{t=1}^{n}\left\{S_{t}^{\mathrm{IO}}\right\} \leq x\right) \longrightarrow \Lambda(x), \quad n \longrightarrow \infty, \\
P\left(\max _{t=1}^{n}\left\{S_{t}^{\mathrm{AO}}\right\} \leq x\right) \rightarrow \Lambda(x), \\
P\left(\max _{t=1}^{n}\left\{C_{t}^{\mathrm{IO}}\right\} \leq x\right) \rightarrow \Lambda(x), \\
P\left(\max _{t=1}^{n}\left\{C_{t}^{\mathrm{AO}}\right\} \leq x\right) \longrightarrow \Lambda(x), \quad n \longrightarrow \infty .
\end{gathered}
$$

If there are several outliers, the main idea is to first detect the maximum outlier by using our method and to obtain new test statistics by deleting its effect. Then, we go on to detect the next outlier and repeat the procedure till there is no outlier. When two types of outliers appear, the test is

$$
\begin{array}{r}
H_{0}: \omega_{t}^{\mathrm{IO}}=0, \quad \omega_{t}^{\mathrm{AO}}=0 \quad \text { versus } H_{1}: \omega_{t}^{\mathrm{IO}} \neq 0 \quad \text { or } \quad \omega_{t}^{\mathrm{AO}} \neq 0, \\
t=1, \ldots, n .
\end{array}
$$

For details, we provide the following steps using absolute value test statistics to detect the $\mathrm{AO}$ and IO in AFAR model as follows: (a) Let test statistics $T_{1}=\max _{t=1}^{n}\left\{A_{t}^{\mathrm{IO}}\right\}$ and $T_{2}=$ $\max _{t=1}^{n}\left\{A_{t}^{\mathrm{AO}}\right\}$. (b) Calculate $\hat{\lambda}_{t}^{\mathrm{IO}}$ and $\hat{\lambda}_{t}^{\mathrm{AO}}$ at every time point, and calculate the maximum $t_{1}=A_{q_{1}}^{\mathrm{IO}}=\max _{t=1}^{n}\left\{A_{t}^{\mathrm{IO}}\right\}$ and $t_{2}=$ $A_{q_{2}}^{\mathrm{AO}}=\max _{t=1}^{n}\left\{A_{t}^{\mathrm{AO}}\right\}$ of absolute value test statistics. (c) Let $C V=\Lambda^{-1}(1-\alpha)=-\log (-\log (1-\alpha))$, if $t_{1}=\max \left\{t_{1}, t_{2}\right\}>$ $C V$, then we believe that the observation is an IO at $t=q_{1}$, and if $t_{2}=\max \left\{t_{1}, t_{2}\right\}>C V$, then we believe that the observation is an $\mathrm{AO}$ at $t=q_{2}$; else, we believe there is no IO or AO. (d) Calculate the $p$-value $p_{1}=P_{H_{0}}\left(T_{1} \geq t_{1}\right)=$ $1-\Lambda\left(t_{1}\right)$ and $p_{2}=P_{H_{0}}\left(T_{2} \geq t_{2}\right)=1-\Lambda\left(t_{2}\right)$, and if $p_{1} \leq \alpha$ or $p_{2} \leq \alpha$, then reject the hypothesis $H_{0}$, believing there is an IO or an AO. Furthermore, we decide whether it is an IO or an AO by its minimal $p$-value. (e) Delete the effect of detected outliers and detect the next outlier. Repeat the above steps till there is no outlier.

\section{Simulation Studies}

Example 8. In this simulation, we consider the following model, where there is only one IO. The sample sizes are 


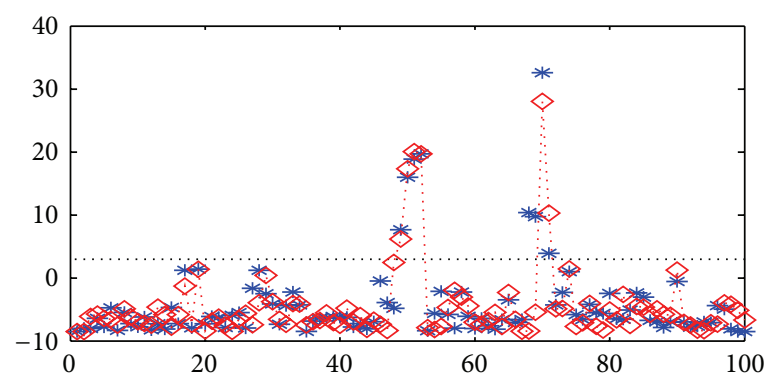

(a)

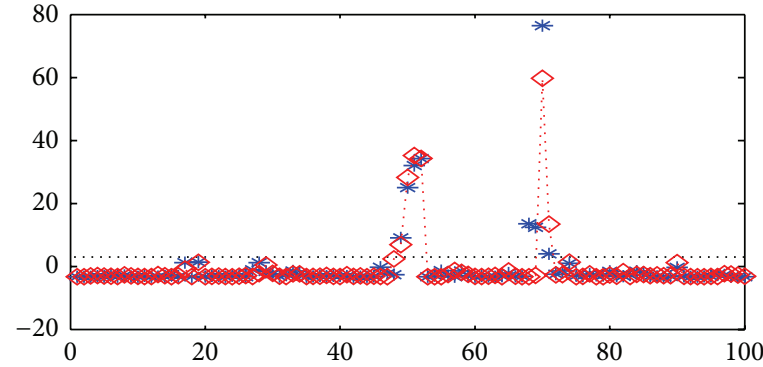

(b)

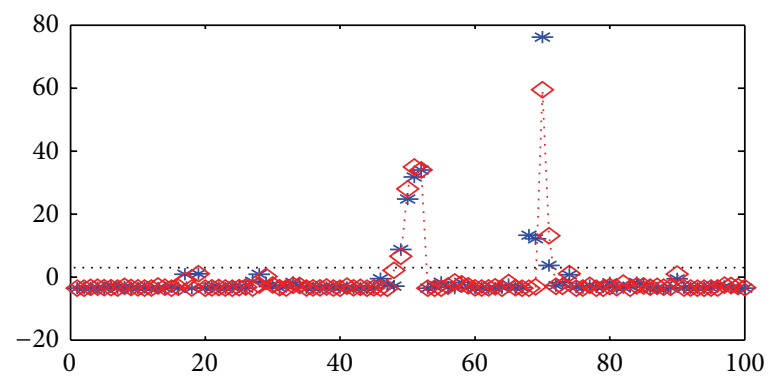

(c)

FIgURE 1: Example 9, first detection.

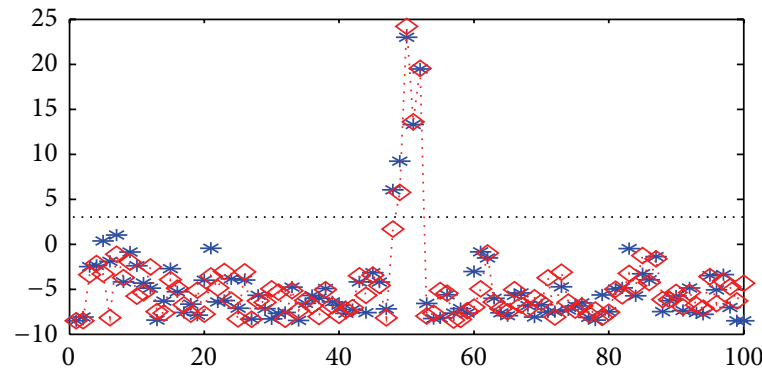

(a)

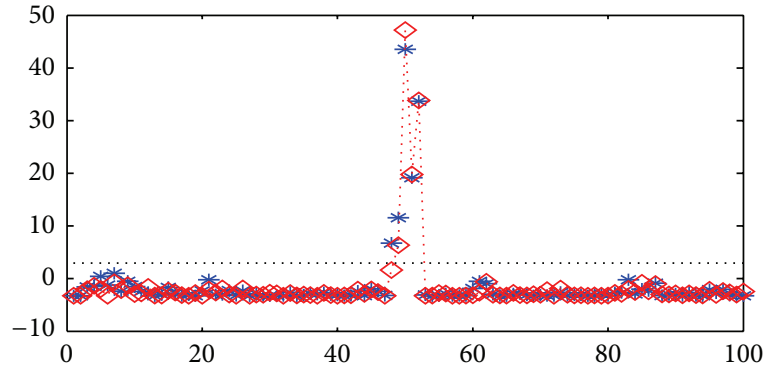

(b)

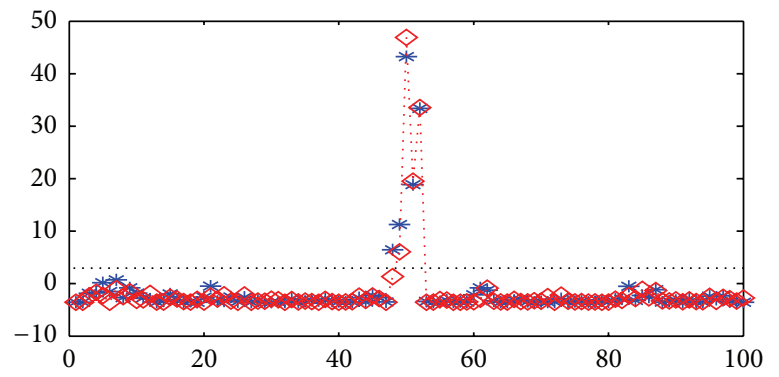

(c)

Figure 2: Example 9, second detection.

$n=50,100,500,1000$, respectively and $\omega_{n / 2}^{\mathrm{IO}}=4$. We have

$$
\begin{gathered}
y_{t}=\frac{\cos \left(1.5 y_{t-2}\right)}{1+y_{t-2}^{2}} y_{t-1}+\left(-\exp \left(-\frac{y_{t-2}^{2}}{2}\right)\right) y_{t-2}+\eta_{t}, \\
\eta_{t}=\varepsilon_{t}+\omega_{n / 2}^{\mathrm{IO}} \cdot \delta\left(t-\frac{n}{2}\right), \quad \varepsilon_{t} \sim N(0,0.5) .
\end{gathered}
$$

For $n=50$, All the three test statistics detect an outlier at $t=25$. The $p$-values for believing it is an IO are $p\left(A^{\mathrm{IO}}\right)=$ $4.8414 e-6, p\left(S^{\mathrm{IO}}\right)=1.8326 e-8$, and $p\left(C^{\mathrm{IO}}\right)=3.3739 e-$ 8 , respectively. The $p$-values for believing it is an $\mathrm{AO}$ are $p\left(A^{\mathrm{AO}}\right)=3.1509 e-4, p\left(S^{\mathrm{AO}}\right)=4.7392 e-5$, and $p\left(C^{\mathrm{AO}}\right)=$ $8.7247 e-5$, respectively. Because the $p$-values for believing it is an IO are smaller than the $p$-values for believing it is an AO, we believe it is an IO, and its influence magnitude is 3.1994. 


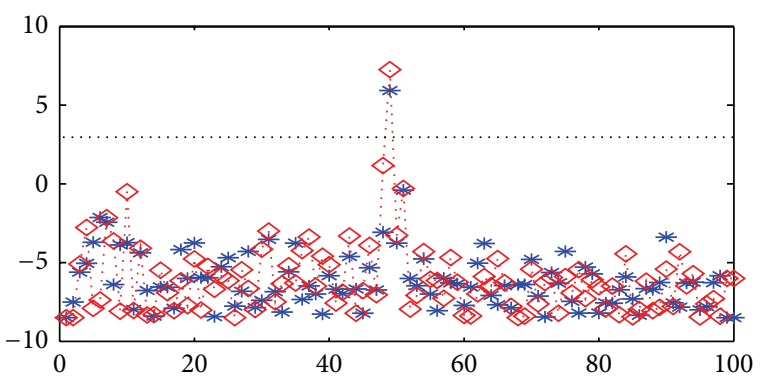

(a)

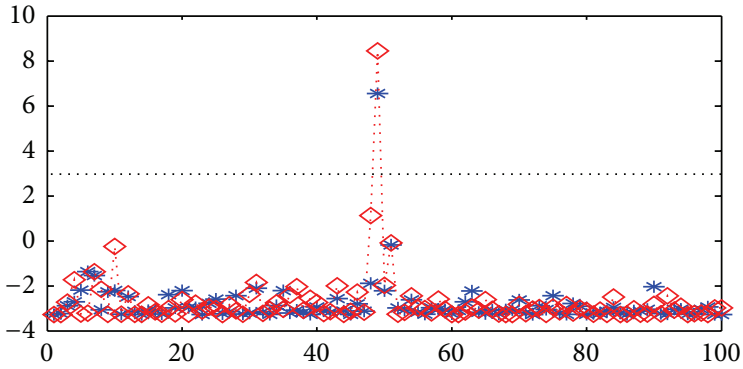

(b)

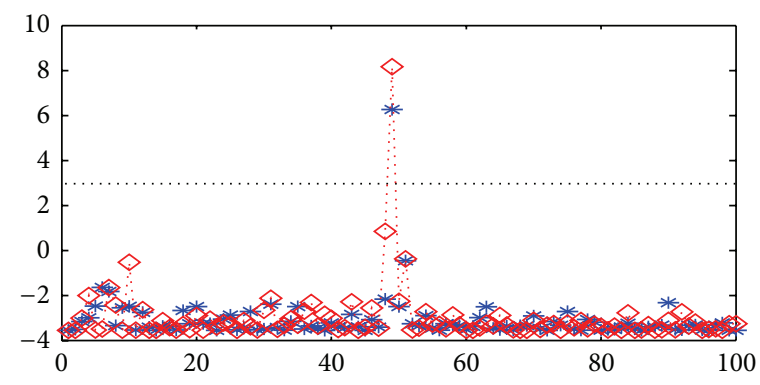

(c)

Figure 3: Example 9, fifth detection.

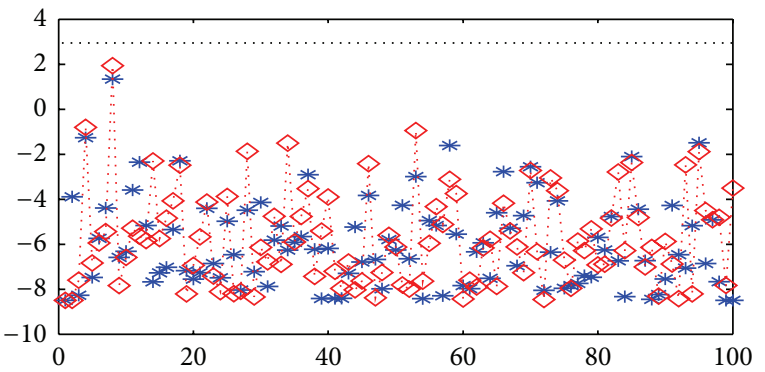

(a)

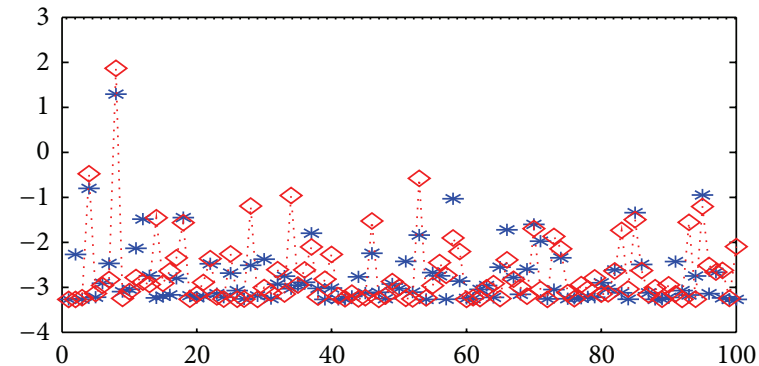

(b)

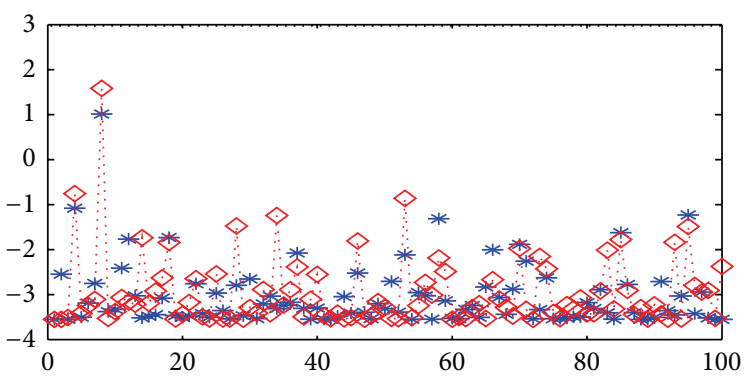

(c)

FIgURE 4: Example 9, sixth detection.

The results are similar for $n=100,500,1000$, respectively, we thus omit the details here. For different test statistics, their $p$ values for believing it is an IO are summarized as follows in Table 1 .

Example 9. There are four IOs and one AO in the time series. These IOs appear at $t=49, t=50, t=51$, and $t=52$ sequentially. Their sizes are 3,6,5, and 4, respectively. One
AO appears at $t=70$ alone, and its size is $7, n=100$. The model is as follows:

$$
\begin{aligned}
x_{t}= & \frac{\cos \left(1.5 x_{t-2}\right)}{1+x_{t-2}^{2}} x_{t-1} \\
& +\left(-\exp \left(-0.5 x_{t-2}^{2}\right)\right) x_{t-2}+\varepsilon_{t}, \quad \varepsilon_{t} \sim N(0,0.6),
\end{aligned}
$$

where $y_{t}$ is obtained by (3) or (4). 
TABLE 1

\begin{tabular}{lccc}
\hline$p$-values & $\begin{array}{c}\text { Absolute value } \\
\text { statistic }\end{array}$ & Square statistic & $\begin{array}{c}\text { Adjusted square } \\
\text { statistic }\end{array}$ \\
\hline$n=50$ & $4.8414 e-6$ & $1.8326 e-8$ & $3.3739 e-8$ \\
$n=100$ & $1.0027 e-6$ & $1.6592 e-9$ & $2.1995 e-9$ \\
$n=500$ & $1.9065 e-6$ & $2.6461 e-8$ & $2.60394 e-8$ \\
$n=1000$ & $2.8048 e-7$ & $1.3358 e-9$ & $1.2590 e-9$ \\
\hline
\end{tabular}

First, we detect an $\mathrm{AO}$ at $t=70$, and its size is 6.8904 . The three test statistics and their corresponding critical values are shown in Figure 1, where Figure 1(a) corresponds to the absolute value test statistics, Figure 1(b) corresponds to square test statistics, and Figure 1(c) corresponds to adjusted square test statistics. Symbol " $\diamond$ ” denotes IO, and “*” denotes AO. Parallel broken line denotes critical value, which is uniform in the figures. Deleting the effect of this $\mathrm{AO}$ and then continuing to detect other outliers in the series, we observe an IO at $t=50$, whose size is 6.0285; see details in Figure 2. Deleting the effect of the aforementioned two outliers and then continuing to detect other outliers in the series, we observe an $\mathrm{IO}$ at $t=51$, whose size is 4.8119 . Deleting the effect of the aforementioned three outliers and then continuing to detect other outliers in the series, we observe an IO at $t=52$, whose size is 4.0024 . Deleting the effect of the aforementioned four outliers and then continuing to detect other outlier in the series, we detect an IO at $t=$ 49, whose size is 2.9046; see Figure 3. Deleting the effect of the aforementioned five outliers and then continuing to detect other outliers in the series, we have not detected any outlier; see Figure 4. Considering the length of paper, we omit some figures here. The result is consistent with the advance enactment.

\section{Conclusions}

The FAR model is mainly featured by the model-dependentvariable, which in one way or another limits the scope of its applications. As a generalization of the class of models, AFAR model clearly covers a larger range of objects than the FAR model, which makes it possible to reduce modeling biases [17] via choosing a proper model-dependent direction. This paper is concerned with detecting $\mathrm{AO}$ and $\mathrm{IO}$ in AFAR models using extreme value methods. We derive the asymptotic distribution of test statistics and provide a control for significance level, which serves as an extension and improvement of existing methods. Based on several simulation studies, we give conclusion remarks as follows. (a) The extreme value method for detecting outliers in AFAR models is tractable and effective not only for IO and AO, but also for separate outliers and outlier patches. Furthermore, it is shown that our method can reduce possible effects of masking and swamping. (b) When applying extreme value theory to detect outliers with a relatively small size samples at hand, the employment of square test statistics works better than that of adjusted square test statistics as well as absolute value test statistics. While following the increments in the samples size, the detecting effect of adjusted square test statistics also increases against that of square test statistics and absolute value test statistics (e.g., see Table 1). (c) Selection of model parameters and the magnitude of outliers have huge influences on the effect of detection.

\section{Acknowledgments}

The authors sincerely wish to thank the editor and the three referees for their insightful suggestions which have led to improving the early version of the paper. The research is supported by the National Natural Science Foundation of China (11171065), the Natural Science Foundation of Jiangsu Province (BK2011058), and Research Fund for the Doctoral Program of Higher Education of China (20120092110021).

\section{References}

[1] C. C. Aggarwal, Outlier Analysis, Springer, New York ,NY, USA, 2013.

[2] X. Jie, M. Li, W. Zhao, and S. Y. Chen, "Bound maxima as a traffic feature under DDOS flood attacks," Mathematical Problems in Engineering, vol. 2012, Article ID 419319, 20 pages, 2012.

[3] M. Li and W. Zhao, "On bandlimitedness and lag-limitedness of fractional Gaussian noise," Physica A, vol. 392, no. 9, pp. 19551961, 2013.

[4] M. Li, Y.-Q. Chen, J.-Y. Li, and W. Zhao, "Hölder scales of sea level," Mathematical Problems in Engineering, Article ID 863707, 22 pages, 2012.

[5] C. W. S. Chen, "Detection of additive outliers in bilinear time series," Computational Statistics \& Data Analysis, vol. 24, no. 3, pp. 283-294, 1997.

[6] M. Li, "Fractal time series-a tutorial review," Mathematical Problems in Engineering, Article ID 157264, 26 pages, 2010.

[7] Z. Cai, J. Fan, and Q. Yao, "Functional-coefficient regression models for nonlinear time series," Journal of the American Statistical Association, vol. 95, no. 451, pp. 941-956, 2000.

[8] F. Battaglia, "Outliers in functional autoregressive time series," Statistics \& Probability Letters, vol. 72, no. 4, pp. 323-332, 2005.

[9] F. Battaglia and L. Orfei, "Outlier detection and estimation in nonlinear time series," Journal of Time Series Analysis, vol. 26, no. 1, pp. 107-121, 2005.

[10] P. Chen, L. Li, Y. Liu, and J.-G. Lin, "Detection of outliers and patches in bilinear time series models," Mathematical Problems in Engineering, Article ID 580583, 10 pages, 2010.

[11] P. Chen, J. Yang, and L. Y. Li, "Synthetic Detection of Change Point and Outliers in Bilinear Time SeriesModels," International Journal of Systems Science. In press.

[12] R. A. Martin, "Extreme value analysis of optimal level-crossing prediction for linear Gaussian processes," Journal of Time Series Analysis, vol. 33, no. 4, pp. 583-607, 2012.

[13] K. Zhu and S. Ling, "Likelihood ratio tests for the structural change of an AR(p) model to a threshold AR(p) model," Journal of Time Series Analysis, vol. 33, no. 2, pp. 223-232, 2012.

[14] P. Chareka, F. Matarise, and R. Turner, "A test for additive outliers applicable to long-memory time series," Journal of Economic Dynamics \& Control, vol. 30, no. 4, pp. 595-621, 2006.

[15] W.-K. Fung, Z.-Y. Zhu, B.-C. Wei, and X. He, "Influence diagnostics and outlier tests for semiparametric mixed models," Journal of the Royal Statistical Society B, vol. 64, no. 3, pp. 565579, 2002. 
[16] A. M. Río, Extreme value theory-based P values in time series outlier detection [Ph.D. thesis], University of Wisconsin Madison, 2005.

[17] J. Fan and Q. Yao, Nonlinear time series, Springer Series in Statistics, Springer, New York, NY, USA, 2003.

[18] M. R. Leadbetter and H. Rootzén, "Extremal theory for stochastic processes," The Annals of Probability, vol. 16, no. 2, pp. 431$478,1988$. 


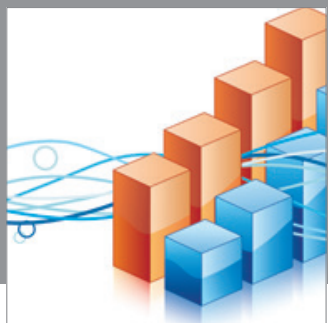

Advances in

Operations Research

mansans

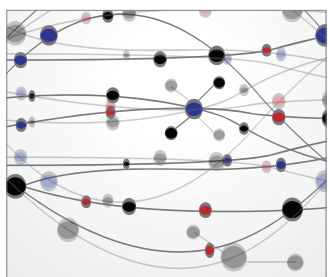

The Scientific World Journal
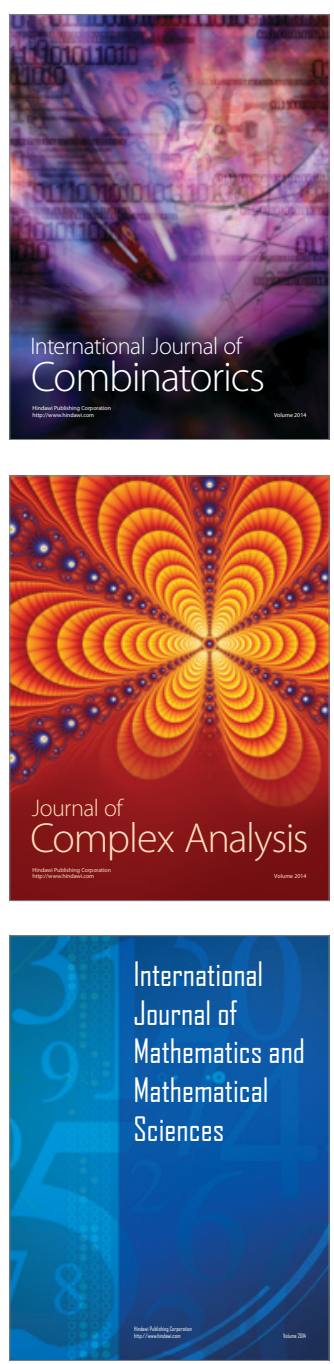
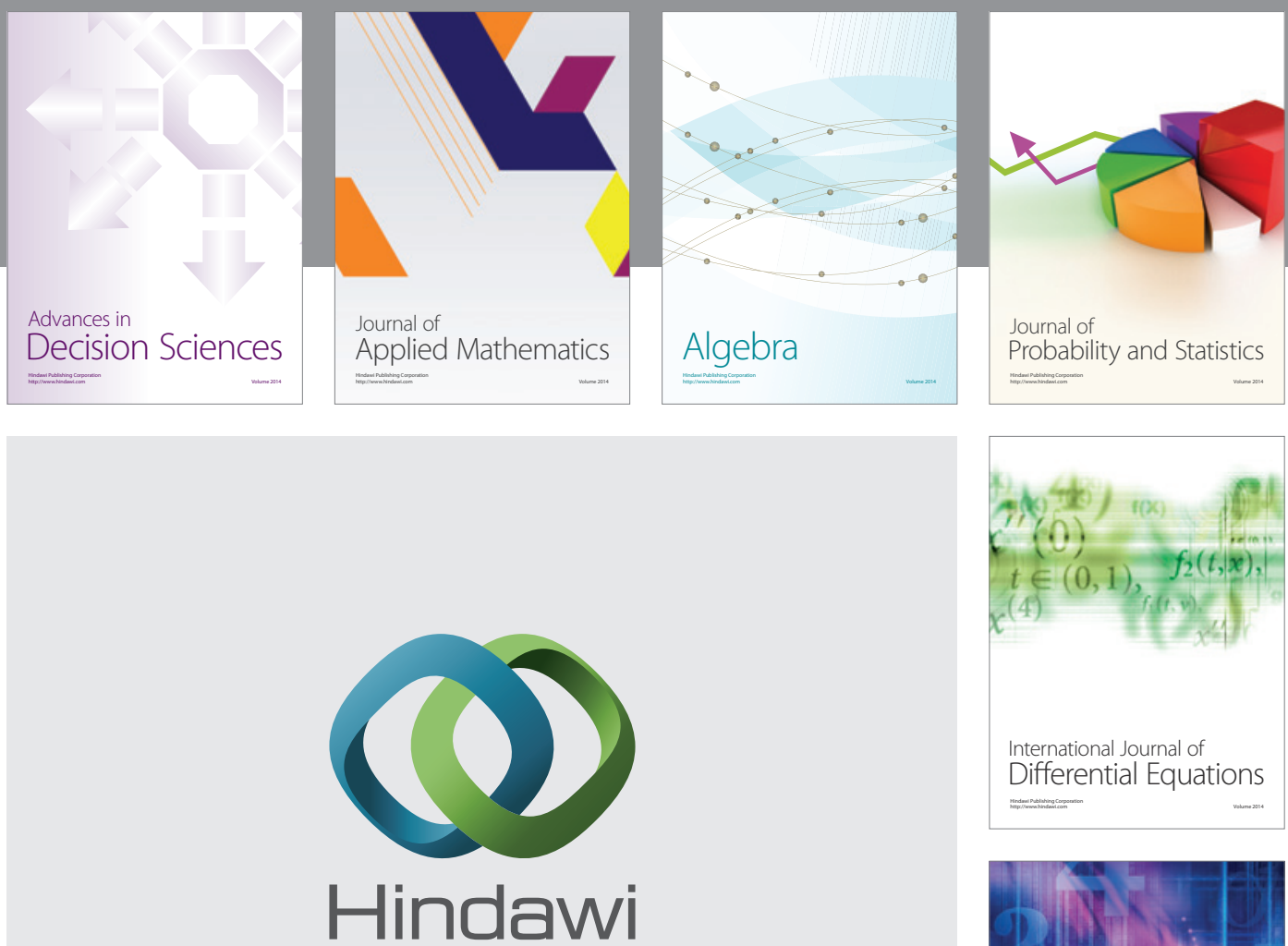

Submit your manuscripts at http://www.hindawi.com
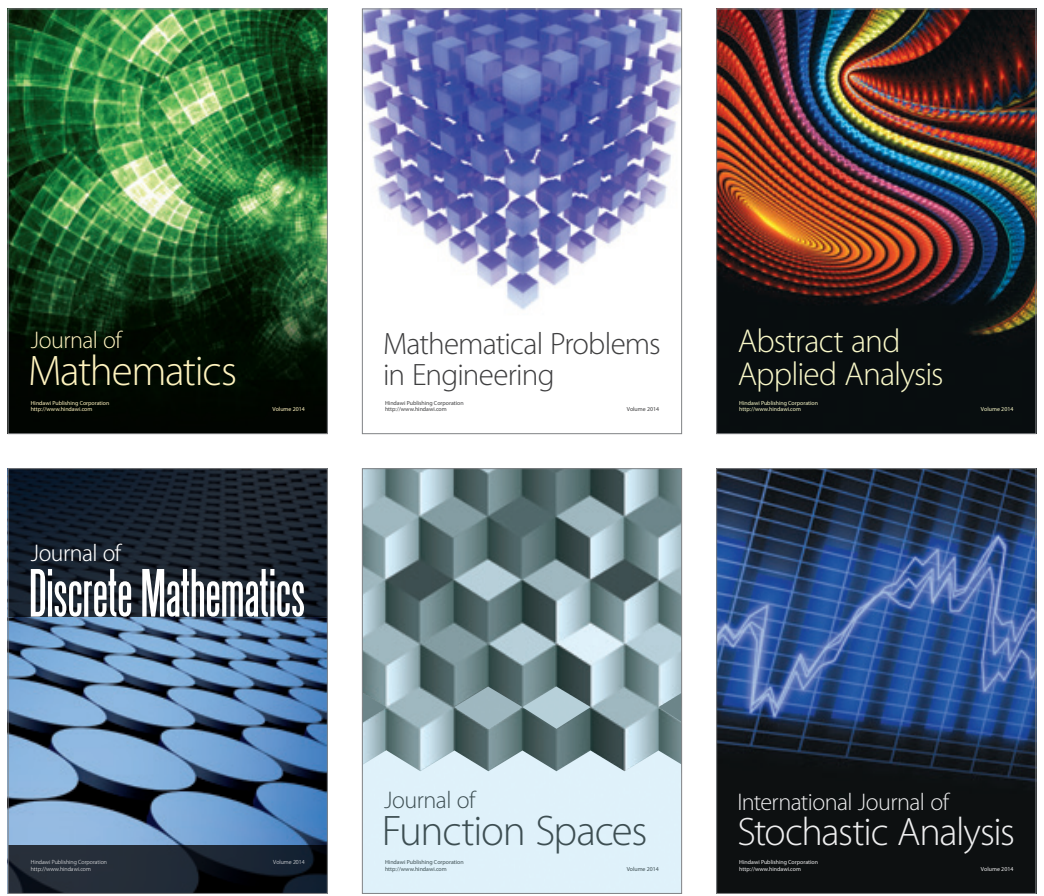

Journal of

Function Spaces

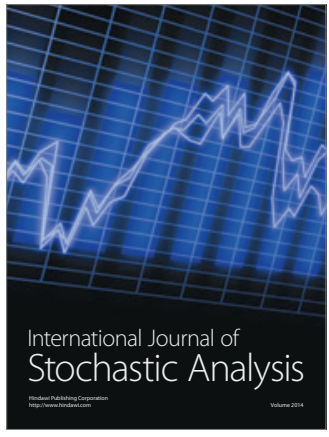

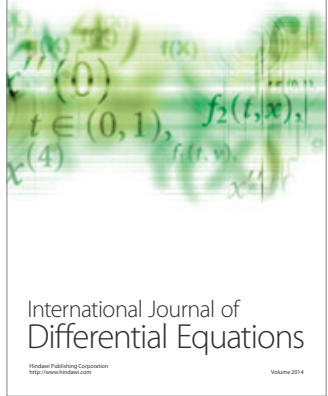
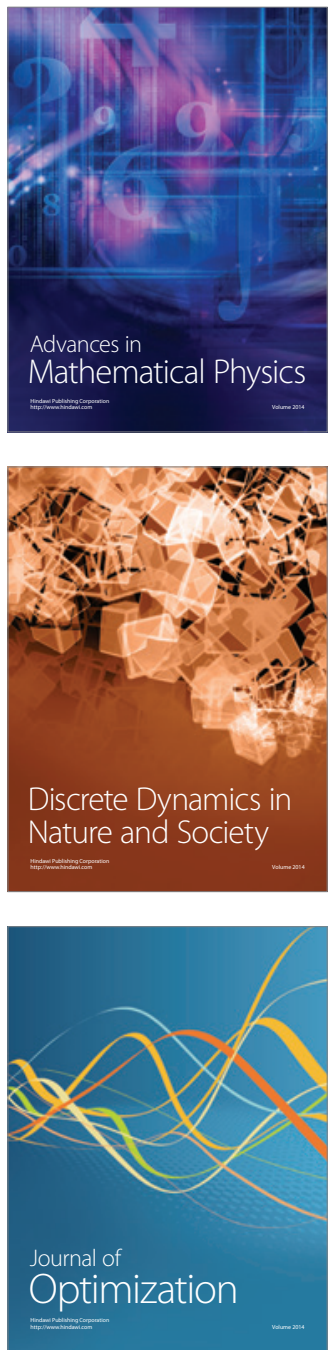